\title{
Numerical Study of Two-Dimensional Volterra Integral Equations by RDTM and Comparison with DTM
}

\author{
Reza Abazari' ${ }^{1}$ and Adem Kılıçman ${ }^{2}$ \\ ${ }^{1}$ Young Researchers and Elite Club, Ardabil Branch, Islamic Azad University, Ardabil, Iran \\ ${ }^{2}$ Department of Mathematics and Institute of Mathematical Research, University Putra Malaysia (UPM), 43400 Serdang, Malaysia
}

Correspondence should be addressed to Reza Abazari; abazari-r@uma.ac.ir

Received 17 April 2013; Accepted 10 June 2013

Academic Editor: Santanu Saha Ray

Copyright @ 2013 R. Abazari and A. Kılıçman. This is an open access article distributed under the Creative Commons Attribution License, which permits unrestricted use, distribution, and reproduction in any medium, provided the original work is properly cited.

\begin{abstract}
The two-dimensional Volterra integral equations are solved using more recent semianalytic method, the reduced differential transform method (the so-called RDTM), and compared with the differential transform method (DTM). The concepts of DTM and RDTM are briefly explained, and their application to the two-dimensional Volterra integral equations is studied. The results obtained by DTM and RDTM together are compared with exact solution. As an important result, it is depicted that the RDTM results are more accurate in comparison with those obtained by DTM applied to the same Volterra integral equations. The numerical results reveal that the RDTM is very effective, convenient, and quite accurate compared to the other kind of nonlinear integral equations. It is predicted that the RDTM can be found widely applicable in engineering sciences.
\end{abstract}

\section{Introduction}

Mathematical modeling of many problems in science, engineering, physics, and other disciplines leads to linear and nonlinear integrodifferential equations (IDE). The great use of mathematical models including integrodifferential equations is one of the main reasons obtaining the solutions of this kind of problems (see, e.g., [1-3] and the references therein). So, it is very important to get some information about the analytical solutions of these problems because these solutions give significant information about the character of the modeled event. But, in some cases, it is more difficult to obtain analytical solutions of these models. These are usually difficult to solve analytically, and in many cases the solution must be approximated. To approximate the solutions of these models, in recent years several numerical approaches have been proposed.

In this paper, we consider the following Volterra type of integral equation $[4,5]$ :

$$
u(x, t)-\int_{0}^{t} \int_{0}^{x} K(x, t, y, z, u(y, z)) d y d z=f(x, t)
$$

where $K$ and $f$ are continuous functions and $K$ has the following form:

$$
K(x, t, y, z, u(y, z))=\sum_{i=0}^{m} p_{i}(x, t) q_{i}(y, z, u(y, z)) .
$$

The one-dimensional Volterra type of integral equation has been solved by many numerical methods, such as collocation methods [1], Taylor-series expansion methods [2], Gausstype quadratures method [3], spectral methods [6], Chebyshev polynomial method [7], Tau method [8], sine-cosine wavelets method [9], Monte Carlo method [10], and Haar functions method [11].

But in two-dimensional cases, a small amount of work has been done (see, e.g., [12-14]). Very recently, Tari et al. in [4] employed the classic differential transform method for solving two-dimensional Volterra type of integral equations (1), and Jang in [5] improved the proofs of the presented theorems by Tari et al. in [4]. They derived fundamental properties of the differential transforms of some kernel functions $K$ in Volterra integral equations. 
However, the classic differential transform method, introduced by Zhou [15], is based on the definition of the differential transform, which is a Taylor series. Thus, it requires a cumbersome calculation to obtain the basic properties of the differential transforms. Some of DTM applications are mentioned in [16-21].

Recently, Keskin and Oturanç introduced a reduced form of DTM as reduced DTM (RDTM) and applied it to approximate some PDE [22] and factional PDEs [23]. More recently, Abazari and Ganji [24] extended RDTM to study the partial differential equation with proportional delay in $t$ and shrinking in $x$ and showed that, as a special advantage of RDTM rather than DTM, the reduced differential transform recursive equations produce exactly all the Poisson series coefficients of solutions based on the initial condition as weighted function, whereas the differential transform recursive equations produce exactly all the Taylor series coefficients of solutions.

Here, we suggest the RDTM, for the approximating of the solutions of the two-dimensional Volterra integral equations (1) with the same kernel functions in $[4,5]$. In order to demonstrate the effectiveness of the RDTM, the illustrative examples for the same kernel function of references [5] are presented. These examples show that the RDTM produces exactly all the Poisson series coefficients (see Remark 5) of the exact solutions, whereas, the classic DTM produces exactly all the TAYLOR series coefficients of the exact solutions. As an important result, notwithstanding the simplicity and robustness of RDTM, it is depicted that the RDTM results are more accurate in comparison with those obtained by classic DTM.

\section{Basic Definitions}

With reference to the articles [16-21], the basic definitions of two-dimensional differential transform method (DTM) and their reduced form (RDTM) are introduced in the following two subsections, respectively.

2.1. Two-Dimensional DTM. Consider a function of two variables $w(x, t)$, and suppose that it can be represented as a product of two single-variable functions, that is, $w(x, t)=$ $f(x) g(t)$. On the basis of the properties of the one-dimensional differential transform, the function $w(x, t)$ can be represented as

$$
w(x, t)=\sum_{i=0}^{\infty} F(i) x^{i} \sum_{j=0}^{\infty} G(j) t^{j}=\sum_{i=0}^{\infty} \sum_{j=0}^{\infty} W(i, j) x^{i} t^{j}
$$

where $W(i, j)=F(i) G(j)$ is called the spectrum of $w(x, t)$.

The basic definitions and operations for two-dimensional differential transform are introduced as follows.
Definition 1. If $w(x, t)$ is analytic and continuously differentiable with respect to time $t$ in the domain of interest, then

$$
W(m, n)=\frac{1}{m ! n !}\left[\frac{\partial^{m+n}}{\partial x^{m} \partial t^{n}} w(x, t)\right]_{\substack{x=x_{0} \\ t=t_{0}}}
$$

where the spectrum function $W(m, n)$ is the transformed function, which is also called $T$-function in brief.

The differential inverse transform of $W(k, h)$ is defined as

$$
w(x, t)=\sum_{m=0}^{\infty} \sum_{n=0}^{\infty} W(m, n)\left(x-x_{0}\right)^{m}\left(t-t_{0}\right)^{n} .
$$

Combining (4) and (5), it can be obtained that

$$
\begin{aligned}
w(x, t)= & \sum_{m=0}^{\infty} \sum_{n=0}^{\infty} \frac{1}{m ! n !}\left[\frac{\partial^{m+n}}{\partial x^{m} \partial t^{n}} w(x, t)\right]_{\substack{x=x_{0} \\
t=t_{0}}} \\
& \times\left(x-x_{0}\right)^{m}\left(t-t_{0}\right)^{n}
\end{aligned}
$$

When $\left(x_{0}, t_{0}\right)$ are taken as $(0,0)$, then $(5)$ can be expressed as

$$
w(x, t)=\sum_{m=0}^{\infty} \sum_{n=0}^{\infty} W(m, n) x^{m} t^{n}
$$

In real applications, the function $w(x, t)$ is represented by a finite series of (7) that can be written as

$$
w_{M, N}(x, t)=\sum_{m=0}^{M} \sum_{n=0}^{N} W(m, n) x^{m} t^{n}+R_{M, N}(x, t),
$$

and (7) implies that $R_{M, N}(x, t)=\sum_{m=M+1}^{\infty} \sum_{n=N+1}^{\infty} W(m$, $n) x^{m} t^{n}$ is negligibly small. Usually, the values of $M$ and $N$ are decided by convergency of the series coefficients.

From the above definitions, it can be found that the concept of the two-dimensional differential transform is derived from the two-dimensional Taylor series expansion. With (4) and (5), the fundamental mathematical operations performed using the two-dimensional differential transform may be readily obtained, and these are listed in Table 1. (See $[4,5,15,16]$.

Recently, Jang [5] extended the two-dimensional DTM on (1) as follows.

Theorem 2. Assume that $U(m, n), V(m, n), H(m, n)$, and $G(m, n)$ are the differential transforms of the functions $u(x, t)$, $v(x, t), h(x, t)$, and $g(x, t)$, respectively; then we have the following:

$$
\begin{aligned}
& \text { (a) if } g(x, t)=\int_{0}^{t} \int_{0}^{x} u(y, z) d y d z \text {, then } \\
& \quad G(m, 0)=G(0, n)=0, \quad m, n=0,1, \ldots, \\
& G(m, n)=\frac{1}{m n} U(m-1, n-1), \quad m, n=1,2, \ldots,
\end{aligned}
$$


TABLE 1: The fundamental operations of two-dimensional differential transform method.

\begin{tabular}{lr}
\hline Original function & Transformed function \\
\hline$w(x, t)=u(x, t) \pm v(x, t)$ & $W(m, n)=U(m, n) \pm V(m, n)$ \\
$w(x, t)=c u(x, t)$ & $W(m, n)=c U(m, n)$ \\
$w(x, t)=\frac{\partial}{\partial x} u(x, t)$ & $W(m, n)=(m+1) U(m+1, n)$ \\
$w(x, t)=\frac{\partial}{\partial t} u(x, t)$ & $W(m, n)=(m+1) U(m, n+1)$ \\
$w(x, t)=\frac{\partial^{r+s}}{\partial x^{r} \partial t^{s}} u(x, t)$ & $W(m)=\frac{(m+r) !(n+s) !}{m ! n !} U(m+r, n+s)$ \\
$w(x, t)=u(x, t) v(x, t)$ & $W(m, n)=\sum_{r=0}^{m} \sum_{s=0}^{n} U(r, n-s) V(m-r, s)$ \\
$w(x, t)=x^{\alpha} t^{\beta}$ & $W(m, n)=\delta(m-\alpha, n-\beta)=\left\{\begin{array}{ll}1 \\
0\end{array} \quad\right.$ otherwise \\
\hline
\end{tabular}

(b) if $g(x, t)=\int_{0}^{t} \int_{0}^{x} u(y, z) v(y, z) d y d z$, then

$G(m, 0)=G(0, n)=0, \quad m, n=0,1, \ldots$,

$G(m, n)=\frac{1}{m n} \sum_{\ell=0}^{n-1} \sum_{k=0}^{m-1} U(k, \ell) V(m-k-1, n-\ell-1)$

$m, n=1,2, \ldots$,

(c) if $g(x, t)=h(x, t) \int_{0}^{t} \int_{0}^{x} u(y, z) d y d z$, then

$$
\begin{gathered}
G(m, 0)=G(0, n)=0, \quad m, n=0,1, \ldots, \\
G(m, n)=\frac{1}{m n} \sum_{\ell=0}^{n-1} \sum_{k=0}^{m-1} H(k, \ell) \frac{V(m-k-1, n-\ell-1)}{(m-k)(n-\ell)}, \\
m, n=1,2, \ldots
\end{gathered}
$$

Proof. See [5].

Theorem 3. Assume that $U(m, n), V(m, n)$, and $G(m, n)$ are the differential transforms of the functions $u(x, t), v(x, t)$, and $g(x, t)$, respectively; then we have the following:

(a) if $g(x, t)=\int_{0}^{t} \int_{0}^{x}(u(y, z) / v(y, z)) d y d z$, then

$$
G(m, 0)=G(0, n)=0, \quad m, n=0,1, \ldots,
$$

$$
\begin{aligned}
& \sum_{\ell=0}^{n-1} \sum_{k=0}^{m-1}(m-k+1)(n-\ell+1) V(k, \ell) G(m-k+1, n-\ell+1) \\
& =U(m, n),
\end{aligned}
$$

(b) if $g(x, t)=(1 / v(x, t)) \int_{0}^{t} \int_{0}^{x} u(y, z) d y d z$, then

$$
\begin{gathered}
G(m, 0)=G(0, n)=0, \quad m, n=0,1, \ldots, \\
\sum_{\ell=0}^{n-1} \sum_{k=0}^{m-1} V(k, \ell) G(m-k+1, n-\ell+1) \\
=\frac{1}{(m+1)(n+1)} U(m, n) .
\end{gathered}
$$

Proof. See [5].

2.2. Two-Dimensional Reduced DTM (RDTM). Consider a function of two variables $w(x, t)$, and suppose that it can be represented as a product of two single-variable functions, that is, $w(x, t)=f(x) g(t)$. Based on the properties of onedimensional differential transform, the function $w(x, t)$ can be represented as

$$
w(x, t)=\sum_{i=0}^{\infty} F(i) x^{i} \sum_{j=0}^{\infty} G(j) t^{j}=\sum_{i=0}^{\infty} \sum_{j=0}^{\infty} W(i, j) x^{i} t^{j},
$$

where $W(i, j)=F(i) G(j)$ is called the spectrum of $w(x, t)$.

Remark 4. The poisson function series generates a multivariate Taylor series expansion of the input expression $w$, with respect to the variables $X$, to order $n$, using the variable weights $W$.

Remark 5. The relationship introduced in (14) is the poisson series form of the input expression $w(x, t)$, with respect to the variables $x$ and $t$, to order $N$, using the variable weights $W_{k}(x)$.

Similarly on previous section, the basic definitions of twodifferential reduced differential transformation are introduced as follows. 
Definition 6. If $w(x, t)$ is analytical function in the domain of interest, then the spectrum function

$$
W_{k}(x)=\frac{1}{k !}\left[\frac{\partial^{k}}{\partial t^{k}} w(x, t)\right]_{t=t_{0}}
$$

is the reduced transformed function of $w(x, t)$.

Similarly on previous sections, the lowercase $w(x, t)$ respects the original function while the uppercase $W_{k}(x)$ stands for the reduced transformed function. The differential inverse transform of $W_{k}(x)$ is defined as

$$
w(x, t)=\sum_{k=0}^{\infty} W_{k}(x)\left(t-t_{0}\right)^{k}
$$

Combining (15) and (16), it can be obtained that

$$
w(x, t)=\sum_{k=0}^{\infty} \frac{1}{k !}\left[\frac{\partial^{k}}{\partial t^{k}} w(x, t)\right]_{t=t_{0}}\left(t-t_{0}\right)^{k}
$$

In real applications, the function $w(x, t)$ is represented by a finite series of (16), around $t_{0}=0$, and can be written as

$$
w_{n}(x, t)=\sum_{k=0}^{n} W_{k}(x) t^{k}+R_{n}(x, t)
$$

and (18) implies that $R_{n}(x, t)=\sum_{k=n+1}^{\infty} W_{k}(x) t^{k}$ is negligibly small. Usually, the values of $n$ and $m$ are decided by convergency of the series coefficients. From the above proposition, it can be found that the concept of the reduced two-dimensional differential transform is derived from the two-dimensional differential transform method. With (15) and (16), the fundamental mathematical operations performed by reduced twodimensional differential transform can readily be obtained and listed in Table 2.

Similarly on previous subsection, we can extend the RDTM on Volterra integral equations (1) as follow.

Theorem 7. Assume that $U_{k}(x), V_{k}(x), H_{k}(x)$, and $W_{k}(x)$ are the reduced differential transforms of the functions $u(x, t)$, $v(x, t), h(x, t)$, and $w(x, t)$, respectively; then we have the following:

(a) if $w(x, t)=\int_{t_{0}}^{t} \int_{x_{0}}^{x} u(y, z) v(y, z) d y d z$, then

$$
W_{k}(x)=\frac{1}{k} \int_{x_{0}}^{x}\left(\sum_{r=0}^{k-1} U_{r}(y) V_{k-r-1}(y)\right) d y, \quad k=1,2, \ldots
$$

(b) if $w(x, t)=h(x, t) \int_{t_{0}}^{t} \int_{x_{0}}^{x} u(y, z) d y d z$, then

$$
W_{k}(x)=\sum_{r=0}^{k} \frac{1}{k-r} H_{r}(x) \int_{x_{0}}^{x} U_{k-r-1}(y) d y, \quad k=1,2, \ldots
$$

Proof. (a) According to the fundamental operations of twodimensional RDTM listed in Table 2 and from Leibnitz formula, we get

$$
\begin{aligned}
\frac{\partial^{k}}{\partial t^{k}} w(x, t)= & \frac{\partial^{k}}{\partial t^{k}}\left(\int_{t_{0}}^{t} \int_{x_{0}}^{x} u(y, z) v(y, z) d y d z\right) \\
= & \int_{x_{0}}^{x} \frac{\partial^{k-1}}{\partial t^{k-1}}\{u(y, t) v(y, t)\} d y \\
= & \int_{x_{0}}^{x}\left\{\sum_{r=0}^{k-1}\left(\begin{array}{c}
k-1 \\
r
\end{array}\right) \frac{\partial^{r}}{\partial t^{r}} u(y, t)\right. \\
& \left.\times \frac{\partial^{k-r-1}}{\partial t^{k-r-1}} v(y, t)\right\} d y,
\end{aligned}
$$

therefore

$$
\begin{gathered}
{\left[\frac{\partial^{k}}{\partial t^{k}} w(x, t)\right]_{t=t_{0}}=\int_{x_{0}}^{x}\left\{\sum_{r=0}^{k-1}\left(\begin{array}{c}
k-1 \\
r
\end{array}\right) r !(k-r-1) !\right.} \\
\left.\times U_{r}(y) V_{k-r-1}(y)\right\} d y \\
=(k-1) ! \int_{x_{0}}^{x}\left\{\sum_{r=0}^{k-1} U_{r}(y) V_{k-r-1}(y)\right\} d y,
\end{gathered}
$$

and then, from using (15), for $k=1,2, \ldots$, we get

$$
W_{k}(x)=\frac{1}{k} \int_{x_{0}}^{x}\left\{\sum_{r=0}^{k-1} U_{r}(y) V_{k-r-1}(y)\right\} d y .
$$

(b) Analogous to part (a), we get

$$
\begin{aligned}
\frac{\partial^{k}}{\partial t^{k}} w(x, t) & =\frac{\partial^{k}}{\partial t^{k}}\left(h(x, t) \int_{t_{0}}^{t} \int_{x_{0}}^{x} u(y, z) d y d z\right) \\
& =\sum_{r=0}^{k}\left(\begin{array}{l}
k \\
r
\end{array}\right) \frac{\partial^{r}}{\partial t^{r}} h(x, t) \int_{x_{0}}^{x} \frac{\partial^{k-r-1}}{\partial t^{k-r-1}} u(y, t) d y,
\end{aligned}
$$


TABLE 2: The fundamental operations of two-dimensional RDTM.

Original function

Reduced transformed function

$w(x, t)=u(x, t) \pm v(x, t)$

$W_{k}(x)=U_{k}(x) \pm V_{k}(x)$

$w(x, t)=\frac{\partial}{\partial x} u(x, t)$

$W_{k}(x)=\frac{\partial}{\partial x} U_{k}(x)$

$w(x, t)=\frac{\partial}{\partial t} u(x, t)$

$W_{k}(x)=(k+1) U_{k+1}(x)$

$w(x, t)=\frac{\partial^{r+s}}{\partial x^{r} \partial t^{s}} u(x, t)$

$W_{k}(x)=\frac{(k+s) !}{k !} \frac{\partial^{r}}{\partial x^{r}} U_{k+s}(x)$

$w(x, t)=u(x, t) v(x, t)$

$W_{k}(x)=\sum_{r=0}^{k} U_{r}(x) V_{k-r}(x)$

$w(x, t)=x^{m} t^{n}$

$W_{k}(x)=x^{m} \delta(k-n)= \begin{cases}x^{m} & k=n \\ 0 & \text { otherwise }\end{cases}$

therefore

$$
\begin{aligned}
{\left[\frac{\partial^{k}}{\partial t^{k}} w(x, t)\right]_{t=t_{0}}=} & \sum_{r=0}^{k}\left(\begin{array}{l}
k \\
r
\end{array}\right) r !(k-r-1) ! H_{r}(x) \\
& \times \int_{x_{0}}^{x} U_{k-r-1}(y) d y, \\
= & \sum_{r=0}^{k} \frac{k !}{k-r} H_{r}(x) \int_{x_{0}}^{x} U_{k-r-1}(y) d y,
\end{aligned}
$$

and then from using (15), for $k=1,2, \ldots$, we get

$$
W_{k}(x)=\sum_{r=0}^{k} \frac{1}{k-r} H_{r}(x) \int_{x_{0}}^{x} U_{k-r-1}(y) d y .
$$

Theorem 8. Assume that $U_{k}(x), V_{k}(x)$, and $W_{k}(x)$ are the reduced differential transforms of the functions $u(x, t), v(x, t)$, and $w(x, t)$, respectively; then we have the following:

(a) if $w(x, t)=\int_{t_{0}}^{t} \int_{x_{0}}^{x}(u(y, z) / v(y, z)) d y d z$, then

$$
U_{k}(x)=\sum_{r=0}^{k}(r+1) \frac{\partial W_{r+1}(x)}{\partial x} V_{k-r}(y), \quad k=0,1,2, \ldots,
$$

(b) if $w(x, t)=(1 / v(x, t)) \int_{t_{0}}^{t} \int_{x_{0}}^{x} u(y, z) d y d z$, then

$$
k \sum_{r=0}^{k} W_{r}(x) V_{k-r}(x)=\int_{x_{0}}^{x} U_{k-1}(y) d y, \quad k=1,2, \ldots
$$

Proof. (a) By following the same manner as in the Theorem 7, we get

$$
u(x, t)=\frac{\partial^{2} w(x, t)}{\partial x \partial t} v(x, t)
$$

then

$$
\begin{aligned}
\frac{\partial^{k}}{\partial t^{k}} u(x, t) & =\frac{\partial^{k}}{\partial t^{k}}\left(\frac{\partial^{2} w(x, t)}{\partial x \partial t} v(x, t)\right) \\
& =\sum_{r=0}^{k}\left(\begin{array}{l}
k \\
r
\end{array}\right) \frac{\partial^{r+2}}{\partial x \partial t^{r+1}} w(x, t) \frac{\partial^{k-r}}{\partial t^{k-r}} v(y, t),
\end{aligned}
$$

therefore

$$
k ! U_{k}(x)=\sum_{r=0}^{k}\left(\begin{array}{l}
k \\
r
\end{array}\right)(r+1) !(k-r) ! \frac{\partial W_{r+1}}{\partial x}(x) V_{k-r}(x),
$$

and then from using (15), for $k=0,1,2, \ldots$, we get

$$
U_{k}(x)=\sum_{r=0}^{k}(r+1) \frac{\partial W_{r+1}}{\partial x}(x) V_{k-r}(x) .
$$

(b) Analogous to part (a), we get

$$
w(x, t) v(x, t)=\int_{t_{0}}^{t} \int_{x_{0}}^{x} u(y, z) d y d z
$$

then

$$
\frac{\partial^{k}}{\partial t^{k}}\{w(x, t) v(x, t)\}=\int_{x_{0}}^{x} \frac{\partial^{k-1}}{\partial t^{k-1}} u(y, t) d y,
$$

therefore

$$
\sum_{r=0}^{k}\left(\begin{array}{l}
k \\
r
\end{array}\right) \frac{\partial^{r}}{\partial t^{r}} w(x, t) \frac{\partial^{k-r}}{\partial t^{k-r}} v(x, t)=\int_{x_{0}}^{x} \frac{\partial^{k-1}}{\partial t^{k-1}} u(y, t) d y,
$$


and then from using (15), for $k=1,2, \ldots$, we get

$$
k ! \sum_{r=0}^{k} W_{r}(x) V_{k-r}(x)=(k-1) ! \int_{x_{0}}^{x} U_{k-1}(y) d y,
$$

and therefore

$$
k \sum_{r=0}^{k} W_{r}(x) V_{k-r}(x)=\int_{x_{0}}^{x} U_{k-1}(y) d y .
$$

\section{Numerical Results of DTM and RDTM}

In this section, the reduced differential transform technique is described to solve a class of Volterra integral equations (1) with kernel functions of (2). In order to demonstrate the effectiveness of the RDTM, the illustrative examples for the same kernel function of [5] are presented. In each example, the numerical results of DTM, RDTM, and their comparisons with exact solution are given in separate tables. The results of the test examples show that the RDTM results are more powerful than DTM results.

Example 9. In the first example, consider the following twodimensional Volterra integral equation [5]:

$$
\begin{array}{r}
u(x, t)-\int_{0}^{t} \int_{0}^{x} \frac{u(y, z)}{2+\sin (y+z)} d y d z \\
=(x-t)(4+2 \sin (x+t)-x t) .
\end{array}
$$

(a) DTM: Jang [5] solved this equation by using DTM and obtained the following five-term DTM solution:

$$
\begin{aligned}
u_{5,5}(x, t)= & \left(4 x+2 x^{2}-\frac{x^{4}}{3}\right)+\left(-4-\frac{2 x^{3}}{3}+\frac{x^{5}}{15}\right) t \\
& +\left(-2+\frac{x^{4}}{12}\right) t^{2}+\left(\frac{2 x}{3}-\frac{x^{5}}{180}\right) t^{3} \\
& +\left(\frac{1}{3}-\frac{x^{2}}{12}\right) t^{4}+\left(-\frac{x}{15}+\frac{x^{3}}{180}\right) t^{5}
\end{aligned}
$$

(b) RDTM: from Volterra integral equation (38), it is easy to see that the $u(x, 0)=x(4+2 \sin (x))$, and therefore $\mathrm{RDTM}$ version is

$$
U_{0}(x)=x(4+2 \sin (x))
$$

By applying the RDTM properties listed in Theorem 8 , on Volterra integral equation (38), for $k=0,1,2, \ldots$, we get

$U_{k}(x)$

$$
=\sum_{r=0}^{k}(r+1) \frac{d}{d x}
$$

$$
\begin{aligned}
& \times\left\{U_{r+1}(x)\right. \\
& -\sum_{\ell=0}^{r+1}\left(x \delta_{\ell, 0}-\delta_{\ell, 1}\right) \\
& \times\left[4 \delta_{r+1-\ell, 0}+\frac{2 \sin (x+((r+1-\ell) \pi / 2))}{(r+1-\ell) !}\right. \\
& \left.\left.-x \delta_{r+1-\ell, 1}\right]\right\} \\
& \times\left\{2 \delta_{k-r, 0}+\sin \left(x+\frac{(k-r) \pi}{2}\right)\right\},
\end{aligned}
$$

where $U_{i}(x)$ is the reduced differential transform of $u(x, t)$. After expanding the RDTM recurrence equations (41), with initial value of (40), for $k=0,1,2,3,4$, the first five terms of $U_{k}(x)$ are obtained as follows:

$$
\begin{aligned}
& U_{1}(x)=2 x \cos (x)-4-2 \sin (x), \\
& U_{2}(x)=-x \sin (x)-2 \cos (x), \\
& U_{3}(x)=\sin (x)-\frac{1}{3} x \cos (x), \\
& U_{4}(x)=\frac{1}{3} \cos (x)+\frac{1}{12} x \sin (x), \\
& U_{5}(x)=\frac{1}{60} x \cos (x)-\frac{1}{12} \sin (x) .
\end{aligned}
$$

In the same manner, the rest of the components can be obtained by using the recursive equations (41). Substituting the quantities (41) in (18), the approximation solution of Volterra integral equation (38) in the Poisson series form is

$$
\begin{aligned}
U_{5}(x, t)= & 2 x(2+\sin (x))+(2 x \cos (x)-4-2 \sin (x)) t \\
& +(-x \sin (x)-2 \cos (x)) t^{2}+\left(\sin (x)-\frac{x \cos (x)}{3}\right) t^{3} \\
& +\left(\frac{\cos (x)}{3}+\frac{x \sin (x)}{12}\right) t^{4} \\
& +\left(\frac{x \cos (x)}{60}-\frac{\sin (x)}{12}\right) t^{5},
\end{aligned}
$$

which is the same as the first five terms of the Poisson series of the exact solution $u(x, t)=2(x-t)(2+\sin (x+t))$. 
TABLE 3: Comparisons of the exact solution $u(x, t)=2(x-t)(2+\sin (x+t))$, with $U_{5,5}(x, t)$ obtained by classic DTM [5] and $U_{5}(x, t)$ obtained by RDTM at some test points $(x, t)$ in Example 9 .

\begin{tabular}{|c|c|c|c|c|c|c|}
\hline \multirow{2}{*}{$x$} & \multirow{2}{*}{$t$} & \multirow{2}{*}{$u(x, t)$} & \multicolumn{2}{|c|}{ Classic DTM [5] } & \multicolumn{2}{|c|}{ Reduced DTM } \\
\hline & & & $U_{5,5}(x, t)$ & $\left|u(x, t)-U_{5,5}(x, t)\right|$ & $U_{5}(x, t)$ & $\left|u(x, t)-U_{5}(x, t)\right|$ \\
\hline \multirow{4}{*}{0.2} & 0.1 & +0.4591040413 & +0.4591115320 & $7.4906677321 e-06$ & +0.4591040577 & $1.6393507773 e-08$ \\
\hline & 0.4 & -1.0258569894 & -1.0257575253 & $9.9464024681 e-05$ & -1.0257906614 & $6.6327927673 e-05$ \\
\hline & 0.7 & -2.7833269096 & -2.7813944067 & $1.9325029608 e-03$ & -2.7814532650 & $1.8736446239 e-03$ \\
\hline & 1 & -4.6912625375 & -4.6755840000 & $1.5678537548 e-02$ & -4.6756686352 & $1.5593902330 e-02$ \\
\hline \multirow{4}{*}{0.5} & 0.1 & +2.0517139787 & +2.0516661667 & $4.7812049361 e-05$ & +2.0517139939 & $1.5172903822 e-08$ \\
\hline & 0.4 & +0.5566653819 & +0.5573213333 & $6.5595140784 e-04$ & +0.5567258924 & $6.0510490391 e-05$ \\
\hline & 0.7 & -1.1728156344 & -1.1698785000 & $2.9371343869 e-03$ & -1.1711316158 & $1.6840186272 e-03$ \\
\hline & 1 & -2.9974949866 & -2.9817708333 & $1.5724153271 e-02$ & -2.9836944470 & $1.3800539634 e-02$ \\
\hline \multirow{4}{*}{0.8} & 0.1 & +3.8966576735 & +3.8929643413 & $3.6933321451 e-03$ & +3.8966576865 & $1.3025739598 e-08$ \\
\hline & 0.4 & +2.3456312688 & +2.3439851520 & $1.6461167738 e-03$ & +2.3456822669 & $5.0998086549 e-05$ \\
\hline & 0.7 & +0.5994989973 & +0.6014245867 & $1.9255893459 e-03$ & +0.6008906123 & $1.3916149372 e-03$ \\
\hline & 1 & -1.1895390524 & -1.1754026667 & $1.4136385685 e-02$ & -1.1783733000 & $1.1165752332 e-02$ \\
\hline \multirow{4}{*}{1} & 0.1 & +5.2041732481 & +5.1881855000 & $1.5987748111 e-02$ & +5.2041732592 & $1.1127633925 e-08$ \\
\hline & 0.4 & +3.5825396760 & +3.5680853333 & $1.4454342653 e-02$ & +3.5825824990 & $4.2822997450 e-05$ \\
\hline & 0.7 & +1.7949988863 & +1.7840151667 & $1.0983719605 e-02$ & +1.7961453619 & $1.1464756153 e-03$ \\
\hline & 1 & +0.0000000000 & -0.0000000000 & -0.0000000000 & +0.0090050384 & $9.0050384311 e-03$ \\
\hline
\end{tabular}

The numerical results obtained with RDTM are presented in Table 3, in comparison with the classic DTM solution of [5] and the exact solution $u(x, t)=2(x-t)(2+\sin (x+t))$, for some points of the intervals $0 \leq x \leq 1$ and $0 \leq t \leq 1$.

Example 10. In the second example, consider the following two-dimensional Volterra integral equation [5]:

$$
\begin{aligned}
u(x, t) & -2 \int_{0}^{t} \int_{0}^{x} e^{y-z} u(y, z) d y d z \\
= & \sin (x+t)\left(e^{x-t}+1\right)-e^{-t} \sin (t)-e^{x} \sin (x)
\end{aligned}
$$

(a) DTM: the approximation solution of this equation is also obtained by DTM in [5] as follows:

$$
\begin{aligned}
u_{5,5}(x, t)= & \left(x-\frac{x^{3}}{6}+\frac{x^{5}}{120}\right)+\left(1-\frac{x^{2}}{2}+\frac{x^{4}}{24}\right) t \\
& +\left(-\frac{x}{2}+\frac{x^{3}}{24}-\frac{x^{5}}{240}\right) t^{2}+\left(-\frac{1}{6}+\frac{x^{2}}{12}-\frac{x^{4}}{144}\right) t^{3} \\
& +\left(\frac{x}{24}-\frac{x^{3}}{144}+\frac{x^{5}}{2880}\right) t^{4} \\
& +\left(\frac{1}{120}-\frac{x^{2}}{240}+\frac{x^{4}}{2880}\right) t^{5} .
\end{aligned}
$$

(b) RDTM: it is easy to see that the $u(x, 0)=\sin (x)$, and therefore RDTM version is

$$
U_{0}(x)=\sin (x)
$$

By applying the RDTM on nonlinear Volterra integral equation (44), for $k=1,2, \ldots$, we get

$$
\begin{aligned}
U_{k}(x)- & \left\{\sum_{r=0}^{k} \frac{\sin (x+r \pi / 2)}{r !}\left(\frac{(-1)^{k-r} e^{x}}{(k-r) !}+\delta_{k-r, 0}\right)\right. \\
& \left.-\sum_{r=0}^{k} \frac{(-1)^{r} \sin ((k-r) \pi / 2)}{r !(k-r) !}-\delta_{k, 0} e^{x} \sin (x)\right\} \\
= & \frac{2}{k} \int_{0}^{x}\left\{\sum_{r=0}^{k-1} e^{y} \frac{(-1)^{r}}{r !} U_{k-r-1}(y)\right\} d y,
\end{aligned}
$$

where $U_{i}(x)$ is the reduced differential transform of $u(x, t)$. After expanding the RDTM recurrence equations (47), with initial value of (46), for $k=1,2,3,4,5$, the first five terms of $U_{k}(x)$ are obtained as follows:

$$
\begin{aligned}
& U_{1}(x)=\cos (x), \\
& U_{2}(x)=-\frac{1}{2} \sin (x),
\end{aligned}
$$


TABLE 4: Comparisons of the exact solution $u(x, t)=\sin (x+t)$, with $U_{5,5}(x, t)$ obtained by classic DTM [5] and $U_{5}(x, t)$ obtained by reduced DTM at some test points $(x, t)$ in Example 10 .

\begin{tabular}{|c|c|c|c|c|c|c|}
\hline \multirow{2}{*}{$x$} & \multirow{2}{*}{$t$} & \multirow{2}{*}{$u(x, t)$} & \multicolumn{2}{|c|}{ Classic DTM [5] } & \multicolumn{2}{|c|}{ Reduced DTM } \\
\hline & & & $U_{5,5}(x, t)$ & $\left|u(x, t)-U_{5,5}(x, t)\right|$ & $U_{5}(x, t)$ & $\left|u(x, t)-U_{5}(x, t)\right|$ \\
\hline \multirow{4}{*}{0.2} & 0.1 & 0.2955202067 & 0.2955202184 & $1.1688660428 e-08$ & 0.2955202070 & $2.9532337686 e-10$ \\
\hline & 0.4 & 0.5646424734 & 0.5646439552 & $1.4818049646 e-06$ & 0.5646439183 & $1.4448767682 e-06$ \\
\hline & 0.7 & 0.7833269096 & 0.7833750550 & $4.8145422517 e-05$ & 0.7833749959 & $4.8086256376 e-05$ \\
\hline & 1 & 0.9320390860 & 0.9325020000 & $4.6291403277 e-04$ & 0.9325019239 & $4.6283789648 e-04$ \\
\hline \multirow{4}{*}{0.5} & 0.1 & 0.5646424734 & 0.5646461680 & $3.6945737146 e-06$ & 0.5646424741 & $6.8315986201 e-10$ \\
\hline & 0.4 & 0.7833269096 & 0.7833397500 & $1.2840372517 e-05$ & 0.7833299139 & $3.0042708277 e-06$ \\
\hline & 0.7 & 0.9320390860 & 0.9321460859 & $1.0699997027 e-04$ & 0.9321309857 & $9.1899716402 e-05$ \\
\hline & 1 & 0.9974949866 & 0.9983398437 & $8.4485714595 e-04$ & 0.9983208230 & $8.2583639762 e-04$ \\
\hline \multirow{4}{*}{0.8} & 0.1 & 0.7833269096 & 0.7834038828 & $7.6973172517 e-05$ & 0.7833269106 & $1.0099717729 e-09$ \\
\hline & 0.4 & 0.9320390860 & 0.9322215424 & $1.8245643277 e-04$ & 0.9320433813 & $4.2953023213 e-06$ \\
\hline & 0.7 & 0.9974949866 & 0.9978859380 & $3.9095139595 e-04$ & 0.9976224907 & $1.2750404846 e-04$ \\
\hline & 1 & 0.9738476309 & 0.9752880000 & $1.4403691218 e-03$ & 0.9749626963 & $1.1150653929 e-03$ \\
\hline \multirow{4}{*}{1} & 0.1 & 0.8912073601 & 0.8915382743 & $3.3091424412 e-04$ & 0.8912073612 & $1.1792198329 e-09$ \\
\hline & 0.4 & 0.9854497300 & 0.9861662222 & $7.1649223376 e-04$ & 0.9854546786 & $4.9486333631 e-06$ \\
\hline & 0.7 & 0.9916648105 & 0.9928385451 & $1.1737346864 e-03$ & 0.9918098802 & $1.4506974814 e-04$ \\
\hline & 1 & 0.9092974268 & 0.9118055556 & $2.5081287299 e-03$ & 0.9105512242 & $1.2537973843 e-03$ \\
\hline
\end{tabular}

$$
\begin{aligned}
& U_{3}(x)=-\frac{1}{6} \cos (x), \\
& U_{4}(x)=\frac{1}{24} \sin (x), \\
& U_{5}(x)=-\frac{1}{120} \cos (x) .
\end{aligned}
$$

In the same manner, the rest of the components were obtained by using the recursive equations (47). Substituting the quantities (48) in (18), the approximation solution of Volterra integral equation (44) in the Poisson series form is

$$
\begin{aligned}
U_{5}(x, t)= & \sin (x)+\cos (x) t-\frac{\sin (x)}{2} t^{2} \\
& -\frac{\cos (x)}{6} t^{3}+\frac{\sin (x)}{24} t^{4}-\frac{\cos (x)}{120} t^{5}
\end{aligned}
$$

which is the same as the first five terms of the Poisson series of the exact solution $u(x, t)=\sin (x+t)$. The numerical results obtained with reduced DTM are presented in Table 4, in comparison with the classic DTM solution of [5] and the exact solution $u(x, t)=\sin (x+t)$, for some points of the intervals $0 \leq x \leq 1$ and $0 \leq t \leq 1$.
Example 11. In the third example, consider the following twodimensional Volterra integral equation [5]:

$$
\begin{aligned}
u(x, t) & -e^{t-x} \int_{0}^{t} \int_{0}^{x} u(y, z) d y d z \\
= & \sinh (x+t)\left(e^{t-x}+1\right)-e^{t-x}(\sinh (x)-\sinh (t)) .
\end{aligned}
$$

(a) DTM: the approximation solution of this equation is also obtained by DTM in [5] as follows

$$
\begin{aligned}
u_{5,5}(x, t)= & \left(-x-\frac{x^{3}}{6}-\frac{x^{5}}{120}\right)+\left(1+\frac{x^{2}}{2}+\frac{x^{4}}{24}\right) t \\
& +\left(-\frac{x}{2}-\frac{x^{3}}{12}-\frac{x^{5}}{240}\right) t^{2}+\left(\frac{1}{6}+\frac{x^{2}}{12}+\frac{x^{4}}{144}\right) t^{3} \\
& +\left(-\frac{x}{24}-\frac{x^{3}}{144}-\frac{x^{5}}{2880}\right) t^{4} \\
& +\left(\frac{1}{120}+\frac{x^{2}}{240}+\frac{x^{4}}{2880}\right) t^{5} .
\end{aligned}
$$

(b) RDTM: it is easy to see that the $u(x, 0)=-\sinh (x)$, and therefore RDTM version is

$$
U_{0}(x)=-\sinh (x) .
$$


TABLE 5: Comparisons of the exact solution $u(x, t)=\sinh (t-x)$, with $U_{5,5}(x, t)$ obtained by classic DTM [5] and $U_{5}(x, t)$ obtained by reduced DTM at some test points $(x, t)$ in Example 11.

\begin{tabular}{|c|c|c|c|c|c|c|}
\hline \multirow{2}{*}{$x$} & \multirow{2}{*}{$t$} & \multirow{2}{*}{$u(x, t)$} & \multicolumn{2}{|c|}{ Classic DTM [5] } & \multicolumn{2}{|c|}{ Reduced DTM } \\
\hline & & & $U_{5,5}(x, t)$ & $\left|u(x, t)-U_{5,5}(x, t)\right|$ & $U_{5}(x, t)$ & $\left|u(x, t)-U_{5}(x, t)\right|$ \\
\hline \multirow{4}{*}{0.2} & 0.1 & -0.1001667500 & -0.1001667561 & $6.0968226301 e-09$ & -0.1001667498 & $2.5944103810 e-10$ \\
\hline & 0.4 & +0.2013360025 & +0.2013367851 & $7.8252557270 e-07$ & +0.2013368189 & $8.1631586063 e-07$ \\
\hline & 0.7 & +0.5210953055 & +0.5211116472 & $1.6341756253 e-05$ & +0.5211117115 & $1.6406043554 e-05$ \\
\hline & 1 & +0.8881059822 & +0.8881853333 & $7.9351145710 e-05$ & +0.8881854339 & $7.9451747271 e-05$ \\
\hline \multirow{4}{*}{0.5} & 0.1 & -0.4107523258 & -0.4107529453 & $6.1950968455 e-07$ & -0.4107523251 & $7.0149602793 e-10$ \\
\hline & 0.4 & -0.1001667500 & -0.1001714167 & $4.6666468226 e-06$ & -0.1001641445 & $2.6055545215 e-06$ \\
\hline & 0.7 & +0.2013360025 & +0.2013887643 & $5.2761781823 e-05$ & +0.2014033478 & $1.7345289013 e-05$ \\
\hline & 1 & +0.5210953055 & +0.5215820313 & $4.8672575625 e-04$ & +0.5216052465 & $5.0994098756 e-04$ \\
\hline \multirow{4}{*}{0.8} & 0.1 & -0.7585837018 & -0.7585783977 & $5.3041062001 e-06$ & -0.7585837006 & $1.2071608158 e-09$ \\
\hline & 0.4 & -0.4107523258 & -0.4108535808 & $1.0125499718 e-04$ & -0.4107476947 & $4.6310571234 e-06$ \\
\hline & 0.7 & -0.1001667500 & -0.1002690359 & $1.4228584682 e-04$ & -0.1000423588 & $1.2439120515 e-04$ \\
\hline & 1 & +0.2013360025 & +0.2019546667 & $6.1866412557 e-04$ & +0.2023226727 & $9.8667016118 e-04$ \\
\hline \multirow{4}{*}{1} & 0.1 & -1.0265167257 & -1.0264561563 & $6.0569458175 e-05$ & -1.0265167241 & $1.6018943949 e-09$ \\
\hline & 0.4 & -0.6366535821 & -0.6370106667 & $3.5708451843 e-04$ & -0.6366473802 & $6.2019846800 e-06$ \\
\hline & 0.7 & -0.3045202934 & -0.3051720521 & $6.5175863619 e-04$ & -0.3043519610 & $1.6833243259 e-04$ \\
\hline & 1 & +0.0000000000 & -0.0000000000 & -0.0000000000 & +0.0013512390 & $1.3512390404 e-03$ \\
\hline
\end{tabular}

By applying the RDTM on nonlinear Volterra integral equation (50), for $k=1,2, \ldots$, we get

$$
\begin{aligned}
\sum_{r=0}^{k}\left\{U_{r}(x)+\frac{\delta_{r, 0}\left(1+e^{-x}\right)}{2}\right. & \\
& \left.+\frac{1}{2 r !}\left(1+(-1)^{r} e^{x}-\left(1+2^{r}\right)\left(e^{-x}+e^{-2 x}\right)\right)\right\}\left\{\frac{(-1)^{k-r}}{(k-r) !} e^{x}\right\} \\
= & \frac{1}{k} \int_{0}^{x} U_{k-r}(y) d y,
\end{aligned}
$$

where $U_{i}(x)$ is the reduced differential transform of $u(x, t)$. After expanding the RDTM recurrence equations (53), with initial value of (52), for $k=1,2,3,4,5$, the first five terms of $U_{k}(x)$ are obtain as follows:

$$
\begin{aligned}
& U_{1}(x)=\cosh (x), \\
& U_{2}(x)=-\frac{1}{2} \sinh (x), \\
& U_{3}(x)=\frac{1}{6} \cosh (x), \\
& U_{4}(x)=\frac{1}{24} \sinh (x), \\
& U_{5}(x)=\frac{1}{120} \cosh (x) .
\end{aligned}
$$

In the same manner, the rest of the components were obtained by using the recursive equations (47). Substituting the quantities (48) in (18), the approximation solution of Volterra integral equation (44) in the Poisson series form is

$$
\begin{aligned}
U_{5}(x, t)= & -\sinh (x)+\cosh (x) t-\frac{\sinh (x)}{2} t^{2} \\
& +\frac{\cosh (x)}{6} t^{3}-\frac{\sinh (x)}{24} t^{4}+\frac{\cosh (x)}{120} t^{5},
\end{aligned}
$$

which is same as the first five terms of the Poisson series of the exact solution $u(x, t)=\sinh (t-x)$. The numerical results obtained with reduced DTM are presented in Table 5, in comparison with the classic DTM solution of [5] and the exact solution $u(x, t)=\sinh (t-x)$, for some points of the intervals $0 \leq x \leq 1$ and $0 \leq t \leq 1$.

\section{Conclusions}

In this study, we presented the definition and operation of both two-dimensional differential transformation method (DTM) and their reduced form, the so-called reduced-DTM (RDTM) for finding the solutions of a class of Volterra integral equations. For illustration purposes, we consider three different examples. It is worth pointing out that both DTM and RDTM have convergence for the solutions; actually, the accuracy of the series solution increases when the number of terms in the series solution is increased. From the computational process of DTM and RDTM, we find that the RDTM is easier to apply. In other words, it is obvious that DTM has very complicated computational process rather than RDTM. 
The RDTM reduces the computational difficulties of the DTM and all the calculations can be made with simple manipulations MATLAB. Actually, as a special advantage of RDTM rather than DTM, the reduced differential transform recursive equations produce exactly all the Poisson series coefficients of solutions, whereas the differential transform recursive equations produce exactly all the Taylor series coefficients of solutions. The reliability of the RDTM and the reduction in the size of computational domain give this method a wider applicability. For small value of $x, t$, in Tables 3,4 , and 5, we find that the RDTM has a smaller error than DTM. Also, for large values of $x$, $t$, we may increase the accuracy of the series solution by computing more terms, which is quite easy using MATLAB.

\section{References}

[1] H. Brunner, Collocation Methods for Volterra Integral and Related Functional Differential Equations, vol. 15 of Cambridge Monographs on Applied and Computational Mathematics, Cambridge University Press, Cambridge, UK, 2004.

[2] P. Huabsomboon, B. Novaprateep, and H. Kaneko, "On Taylorseries expansion methods for the second kind integral equations," Journal of Computational and Applied Mathematics, vol. 234, no. 5, pp. 1466-1472, 2010.

[3] H. Kaneko and Y. Xu, "Gauss-type quadratures for weakly singular integrals and their application to Fredholm integral equations of the second kind," Mathematics of Computation, vol. 62, no. 206, pp. 739-753, 1994.

[4] A. Tari, M. Y. Rahimi, S. Shahmorad, and F. Talati, "Solving a class of two-dimensional linear and nonlinear Volterra integral equations by the differential transform method," Journal of Computational and Applied Mathematics, vol. 228, no. 1, pp. 7076, 2009.

[5] B. Jang, "Comments on "Solving a class of two-dimensional linear and nonlinear Volterra integral equations by the differential transform method'”' Journal of Computational and Applied Mathematics, vol. 233, no. 2, pp. 224-230, 2009.

[6] Y.-J. jiang, "On spectral methods for Volterra-type integrodifferential equations," Journal of Computational and Applied Mathematics, vol. 230, no. 2, pp. 333-340, 2009.

[7] A. Akyüz-Daşcioğlu and M. Sezer, "Chebyshev polynomial solutions of systems of higher-order linear Fredholm-Volterra integro-differential equations," Journal of the Franklin Institute. Engineering and Applied Mathematics, vol. 342, no. 6, pp. 688701, 2005.

[8] S. M. Hosseini and S. Shahmorad, "Numerical solution of a class of integro-differential equations by the tau method with an error estimation," Applied Mathematics and Computation, vol. 136, no. 2-3, pp. 559-570, 2003.

[9] M. T. Kajani, M. Ghasemi, and E. Babolian, "Numerical solution of linear integro-differential equation by using sine-cosine wavelets," Applied Mathematics and Computation, vol. 180, no. 2, pp. 569-574, 2006.

[10] R. Farnoosh and M. Ebrahimi, "Monte Carlo method for solving Fredholm integral equations of the second kind," Applied Mathematics and Computation, vol. 195, no. 1, pp. 309-315, 2008.

[11] K. Maleknejad and F. Mirzaee, "Numerical solution of integrodifferential equations by using rationalized Haar functions method," Kybernetes, vol. 35, no. 10, pp. 1735-1744, 2006.
[12] K. E. Atkinson, The Numerical Solution of Integral Equations of the Second Kind, vol. 4 of Cambridge Monographs on Applied and Computational Mathematics, Cambridge University Press, Cambridge, UK, 1997.

[13] H. Guoqiang and W. Jiong, "Extrapolation of Nystrom solution for two dimensional nonlinear Fredholm integral equations," Journal of Computational and Applied Mathematics, vol. 134, no. 1-2, pp. 259-268, 2001.

[14] G. Han and R. Wang, "Richardson extrapolation of iterated discrete Galerkin solution for two-dimensional Fredholm integral equations," Journal of Computational and Applied Mathematics, vol. 139, no. 1, pp. 49-63, 2002.

[15] J. K. Zhou, Differential Transformation and Its Application for Electrical CircuIts, Huazhong University Press, Wuhan, China, 1986.

[16] R. Abazari and A. Borhanifar, "Numerical study of the solution of the Burgers and coupled Burgers equations by a differential transformation method," Computers \& Mathematics with Applications, vol. 59, no. 8, pp. 2711-2722, 2010.

[17] A. Borhanifar and R. Abazari, "Exact solutions for non-linear Schrödinger equations by differential transformation method," Journal of Applied Mathematics and Computing, vol. 35, no. 1-2, pp. 37-51, 2011.

[18] A. Borhanifar and R. Abazari, "Numerical study of nonlinear Schrödinger and coupled Schrödinger equations by differential transformation method," Optics Communications, vol. 283, no. 10, pp. 2026-2031, 2010.

[19] R. Abazari and M. Abazari, "Numerical simulation of generalized Hirota-Satsuma coupled KdV equation by RDTM and comparison with DTM," Communications in Nonlinear Science and Numerical Simulation, vol. 17, no. 2, pp. 619-629, 2012.

[20] R. Abazari, "Solution of Riccati types matrix differential equations using matrix differential transform method," Journal of Applied Mathematics \& Informatics, vol. 27, pp. 1133-1143, 2009.

[21] R. Abazari and R. Abazari, "Numerical study of some coupled PDEs by using differential transformation method," World Academy of Science, Engineering and Technology, vol. 66, pp. 5259, 2010.

[22] Y. Keskin and G. Oturanç, "Reduced differential transform method for partial differential equations," International Journal of Nonlinear Sciences and Numerical Simulation, vol. 10, no. 6, pp. 741-749, 2009.

[23] Y. Keskin and G. Oturanç, “The reduced differential transform method: a new approach to factional partial differential equations," Nonlinear Science Letters A, vol. 1, pp. 207-217, 2010.

[24] R. Abazari and M. Ganji, "Extended two-dimensional DTM and its application on nonlinear PDEs with proportional delay," International Journal of Computer Mathematics, vol. 88, no. 8, pp. 1749-1762, 2011. 


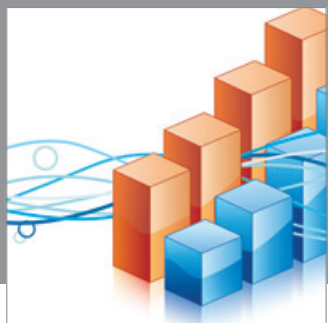

Advances in

Operations Research

mansans

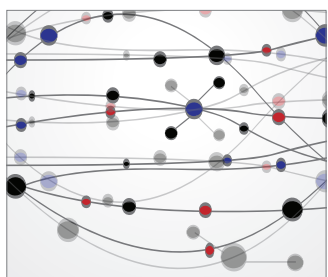

The Scientific World Journal
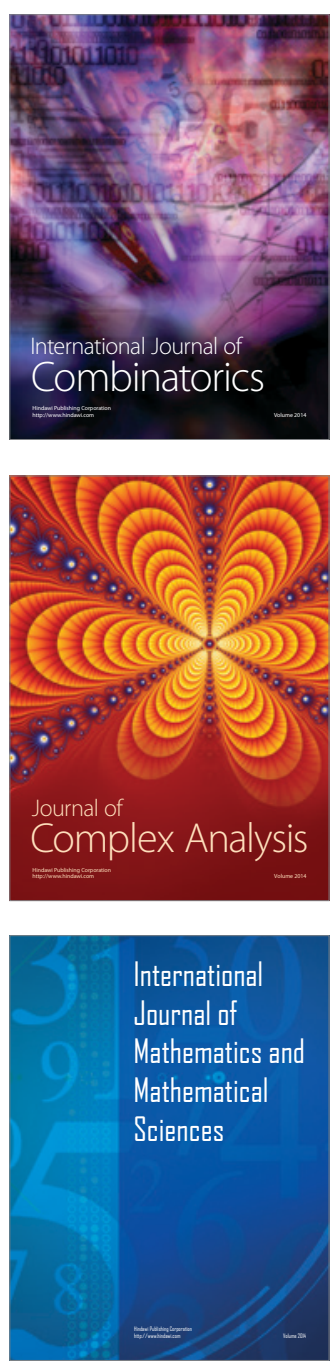
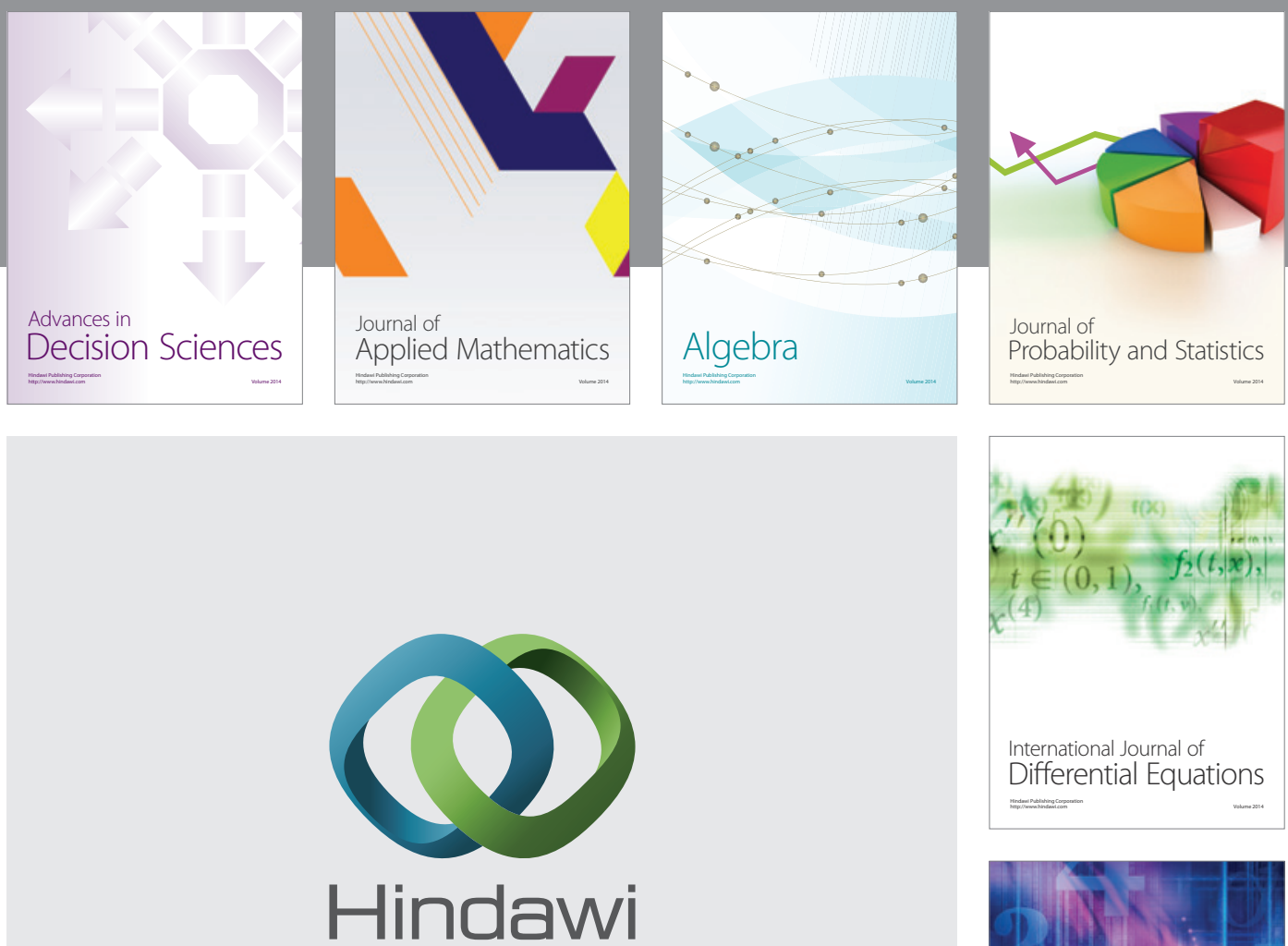

Submit your manuscripts at http://www.hindawi.com
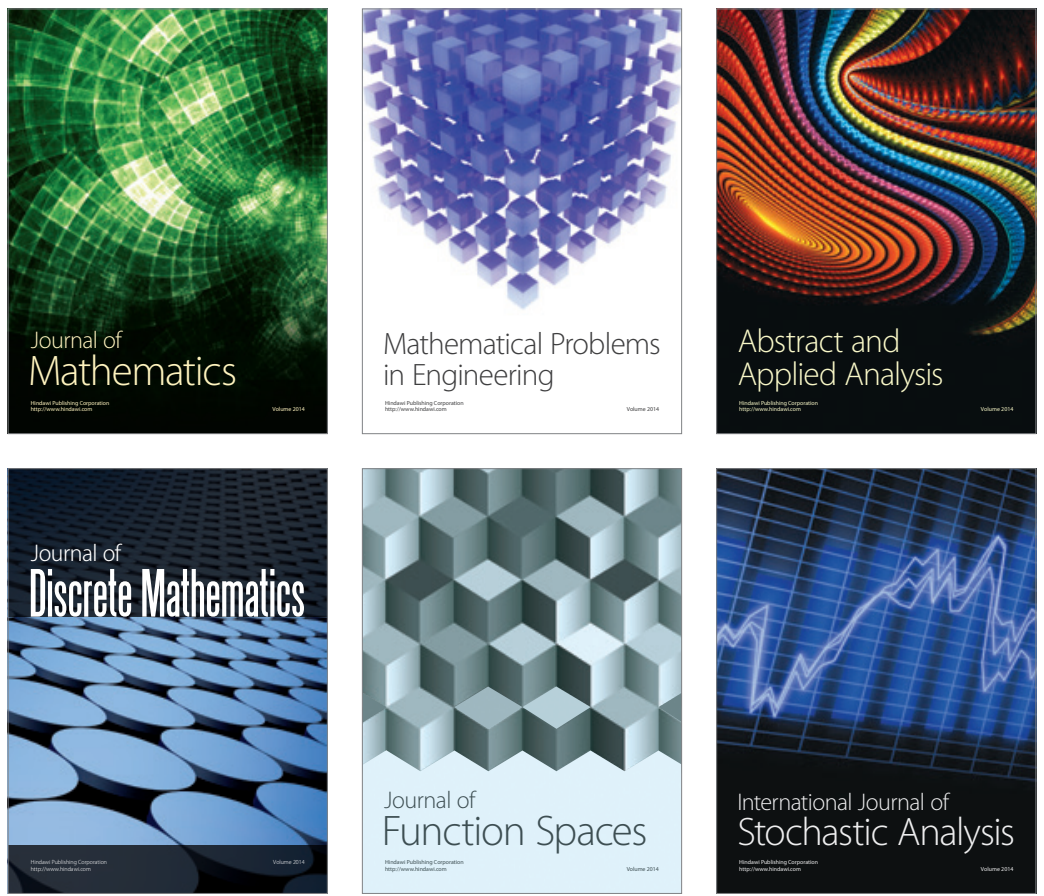

Journal of

Function Spaces

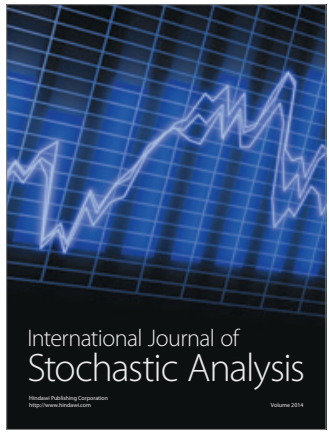

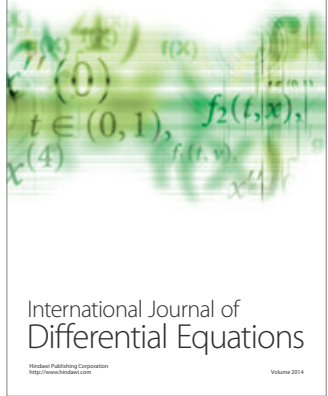
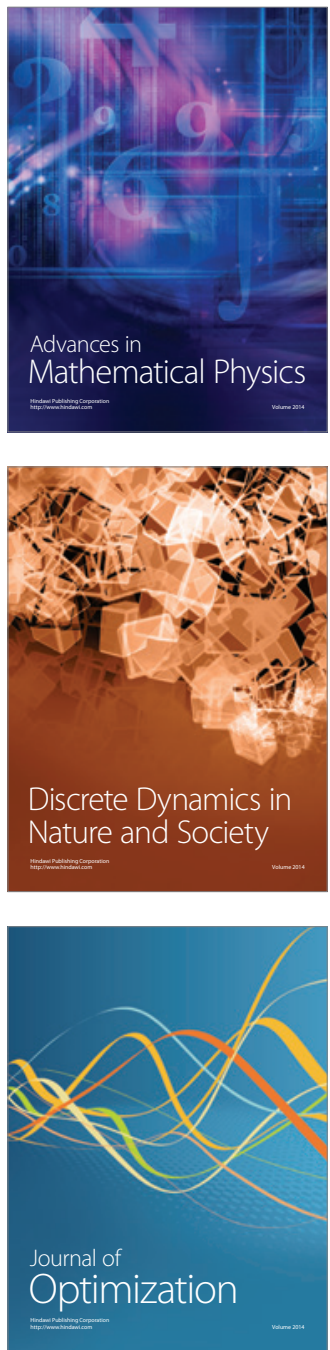